\title{
OTIMIZAÇÃO MULTIOBJETIVO DO DESIGN DE PRODUTOS COM COMPUTAÇÃO EVOLUTIVA: UMA ANÁLISE BIBLIOMÉTRICA E SISTÊMICA
}

Samuel Henrique Werlich (samuelw@alunos.utfpr.edu.br) - PPGEM, UTFPR ${ }^{1}$.

Lucas Iuri dos Santos (lucassantos.1994@alunos.utfpr.edu.br)-PPGEM, UTFPR¹.

Joelton Deonei Gotz (gotz@alunos.utfpr.edu.br) - PPGEM, UTFPR'.

João Felipe Raffs Espolador (joaoe@ alunos.utfpr.edu.br) - PPGEM, UTFPR ${ }^{1}$.

Milton Borsato (borsato@utfpr.edu.br) - PPGEM, UTFPR ${ }^{1}$.

\section{RESUMO}

Nas etapas do projeto informacional e conceitual, do processo de desenvolvimento de produto (PDP), verifica-se que o uso combinado de técnicas de otimização multiobjetivo e de computação evolucionária (CE) podem reduzir significativamente o tempo de desenvolvimento do produto e economia de custos em comparação com as abordagens tradicionais. Este artigo tem como objetivo apresentar uma análise bibliométrica e sistêmica dos principais artigos sobre o uso da otimização multiobjetivo e CE no design de produtos. Para isso, foi utilizado o Knowledge Development Process-Constructivist (ProKnow-C), para a seleção e análise de artigos das principais bases de dados internacionais dos últimos cinco anos. A partir de 1.748 artigos brutos chegou-se a um portfólio bibliográfico de 32 artigos. Foi então feita uma análise bibliométrica avaliando o grau de relevância dos periódicos, o reconhecimento científico dos artigos e as palavras-chave mais utilizadas; e também uma análise sistêmica. As principais tendências encontradas foram o uso do algoritmo genético de classificação por não dominância II (NSGA-II), de algoritmos genéticos multiobjetivo (MOGA) e algoritmos genéticos clássicos. Verificou-se que existem oportunidades de aplicar os métodos utilizados nestas pesquisas em outros sistemas e de incorporar de novos parâmetros de otimização aos métodos utilizados.

Palavras-chave: processo de desenvolvimento de produto; projeto informacional; projeto conceitual; otimização multiobjetivo; computação evolucionária.

\footnotetext{
1 Programa de Pós-graduação em Engenharia Mecânica e Materiais (PPGEM) da Universidade Tecnológica Federal do Paraná (UTFPR).
} 


\section{INTRODUÇÃO}

O avanço dos recursos computacionais e das tecnologias da informação e comunicação desempenham um papel significativo no processo de desenvolvimento de produtos (PDP). O uso da inteligência artificial (IA) vêm desencadeando rápido desenvolvimento de novas tecnologias e métodos, pelos quais proporcionam oportunidades de novos modelos, meios e formas de fabricação, arquiteturas e sistemas de tecnologia para manufatura inteligente, com base na integração com a tecnologia IA (LI et al., 2017).

Em 1956, durante uma conferência no Darthmouth College, o cientista da computação John McCarthy usou pela primeira vez a expressão "inteligência artificial", o que é considerado o marco inicial da IA. Já o termo inteligência computacional (IC) surge na década de 90 como uma subdivisão da IA incluindo abordagens, como: redes neurais artificiais; computação evolutiva (algoritmos genéticos, programação evolutiva, programação genética, sistemas classificadores e estratégia evolutiva); sistemas nebulosos; raciocínio probabilístico; e sistemas híbridos inteligentes (combinações de redes neurais, sistemas nebulosos e computação evolutiva). A computação evolutiva (CE) compreende um conjunto de técnicas de busca e otimização inspiradas na teoria da seleção natural das espécies, publicada por Charles Darwin em 1856 (BÄCK et al., 1997; COELHO \& MARIANI, 2006; GASPARCUNHA, TAKAHASHI \& ANTUNES, 2012; SICHMAN, 2021).

O uso de algoritmos de otimização baseados em CE têm sido tipicamente objeto de estudo da ciência da computação e da matemática, e a sua introdução no campo da engenharia é relativamente nova. No caso de desenvolvimento de produtos, pesquisas de otimização baseados em simulação computacional são realizadas para achar o "design ideal" de um produto nas fases iniciais do desenvolvimento. Para Rozenfeld et al., (2012) o PDP pode ser dividido em três macro fases: pré-lançamento, desenvolvimento e pós-lançamento; e por sua vez, a macro fase de desenvolvimento se divide em: projeto informacional, projeto conceitual, projeto detalhado, preparação da produção e lançamento do produto.

É na fase de projeto informacional que é feito o levantamento das necessidades dos clientes, as quais posteriormente são transformadas em requisitos de projeto. Nesta fase, também são estabelecidos valores e parâmetros para estes requisitos e definidas as especificações-meta. As especificações-meta são um conjunto de objetivos ou metas que o produto deve atender. Estas especificações podem ser vistas como um conjunto de informações, as quais constituem a base para a realização do projeto conceitual. Já na fase de projeto conceitual ocorre a geração 
das alternativas de concepção do produto, por meio da busca, criação, representação e a seleção de soluções com a melhor concepção (ROZENFELD et al., 2012).

Este trabalho trará um recorte de estudos que utilizam técnicas $\mathrm{CE}$ associadas com a otimização multiobjetivo para o design de produtos durante as fases do projeto informacional e conceitual do PDP. Assim, este artigo irá identificar os principais artigos que tratam do assunto e fazer uma análise bibliométrica e sistêmica, através de um processo científico denominado de Knowledge Development Process-Constructivist (ProKnow-C). A Seção 2 apresenta os aspectos metodológicos adotados para o desenvolvimento do trabalho. Já a Seção 3 descreve como foi realizada a seleção do portfólio bibliográfico. Na sequência, as Seções 4 e 5 apresentam respectivamente as análises bibliométrica e sistêmica realizadas. $\mathrm{O}$ trabalho finaliza com as conclusões, apresentando as tendências e oportunidades; e as limitações desta pesquisa.

\section{ASPECTOS METODOLÓGICOS}

O objetivo desta seção é apresentar o procedimento metodológico utilizado para se chegar ao estado da arte sobre o tema. Como mencionado anteriormente, para este estudo adotou-se o ProKnowC, um procedimento que auxilia os pesquisadores a encontrarem os conteúdos mais expressivos dentro de uma extensa quantidade de informações na literatura (ENSSLIN et al., 2010).

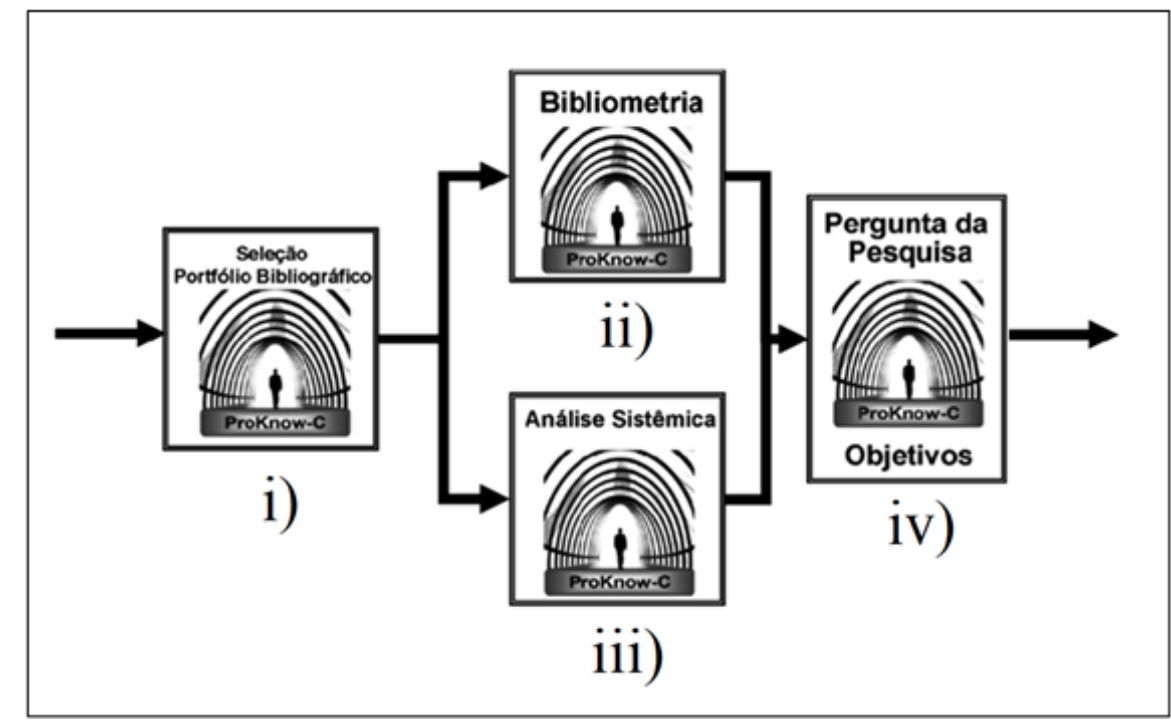

FIGURA 2.1 - Macro-etapas do ProKnow-C. Fonte: Adaptado de Ensslin et al. (2010).

Conforme verifica-se na Figura 2.1 o ProKnow-C é dividido em quatro etapas. A primeira etapa (i), consiste na construção do portfólio bibliográfico, ou seja, a seleção de um banco de artigos e na filtragem destes até encontrar uma amostra representativa. Na segunda etapa (ii) é 
feita a análise bibliométrica do portfólio, na qual ocorre uma análise de quais são os periódicos e as palavras-chave mais recorrentes, além do número de vezes que cada artigo foi citado na literatura com o propósito de se identificar as publicações mais relevantes para o tema de pesquisa. Na terceira etapa (iii), análise sistêmica, é realizada a leitura integral do portfólio resultante, a fim de identificar os problemas de pesquisa, as soluções propostas pelos autores, as lacunas e oportunidades de pesquisa observadas. Por fim, a quarta e última etapa (iv) do ProKnow-C é a definição da pergunta e dos objetivos de pesquisa (ENSSLIN et al., 2010). Este estudo limitou-se nas três primeiras etapas do processo, não seguindo para a elaboração de uma pergunta de pesquisa e de objetivos para um trabalho futuro.

\section{SELEÇÃO DO PORTFÓLIO BIBLIOGRÁFICO}

A seguir apresenta-se como foi feita a seleção do portfólio bibliográfico de acordo com a metodologia do ProKnow-C.

\subsection{Seleção da base de artigos bruta}

A seleção da base de artigos bruta iniciou com a definição do eixo de pesquisa, que foram: "Evolutionary computation", "Product design" e "Multiobjective optimization". Em seguida buscou-se relacionar as palavras-chave (PC's) de por eixo. No Quadro 3.1 pode-se verificar as combinações das PC's usadas para as buscas dos artigos.

QUADRO 3.1 - Combinações das palavras-chave. Fonte: Autoria própria

\begin{tabular}{|c|}
\hline Evolutionary computation \\
\hline Genetic algorithm \\
\hline Evolutionary algorithm \\
\hline Genetic programming \\
\hline Metaheuristic* \\
\hline Meta heuristic* \\
\hline
\end{tabular}

\begin{tabular}{|c|}
\hline Design \\
\hline Product design \\
\hline Product development \\
\hline Computer Aided Design \\
\hline Quality function deployment \\
\hline Finite element method \\
\hline
\end{tabular}

\begin{tabular}{|c|}
\hline Multiobjective optimization \\
\hline Multi-objective optimization \\
\hline Design optimization \\
\hline Optimization \\
\hline
\end{tabular}

O primeiro eixo, composto por 6 PC's, foi combinado com o segundo eixo composto por 6 PC's e com o terceiro eixo com 4 PC's, gerando 144 combinações diferentes através do produto da multiplicação entre os três eixos. A pesquisa foi limitada por artigos de periódicos publicados em língua inglesa entre 2017 e 2021 e ocorreu em três Bases de Dados (BD’s): Scopus, Web of Science e Compendex. Na Tabela 3.1 verifica-se a sintaxe de busca realizada nas BD's no dia 18 de maio de 2021. Nas 3 BD's foram obtidos 1.748 artigos brutos, que compõem o chamado Banco de Artigos Brutos Preliminar. 
TABELA 3.1 - Resultados das sintaxes de busca. Fonte: Autoria própria.

\begin{tabular}{|c|c|c|}
\hline Base de Dados & Sintaxe de Busca & Resultados \\
\hline Compendex & $\begin{array}{l}((((\text { "Evolutionary computation" OR "Genetic algorithms" OR "Evolutionary } \\
\text { algorithms" OR "Genetic programming" OR "Heuristics algorithms" )) WN ALL) } \\
\text { AND ((( "Product design" OR "Product development" OR "Computer Aided } \\
\text { Design" OR "Quality function deployment" OR "Fin ite element method" )) WN } \\
\text { ALL)) AND ((( "Multiobjective optimization" OR "Multi-objective optimization" } \\
\text { OR "Design optimization" )) WN ALL)) AND (JA WN DT) AND (English WN LA) }\end{array}$ & 234 \\
\hline Scopus & $\begin{array}{l}\text { TITLE-ABS-KEY-AUTH ( "Evolutionary computation" OR "Genetic algorithms" } \\
\text { OR "Evolutionary algorithms" OR "Genetic programming" OR "Heuristics } \\
\text { algorithms") AND TITLE-ABS-KEY-AUTH ( "Product design" OR "Product } \\
\text { development" OR "Computer Aided Design" OR "Quality function } \\
\text { deployment" OR "Finite element method") AND TITLE-ABS-KEY-AUTH ( } \\
\text { "Multiobjective optimization" OR "Multi-objective optimization" OR "Design } \\
\text { optimization") AND ( LIMIT-TO (SRCTYPE, "j" )) AND ( LIMIT-TO (DOCTYPE } \\
\text { "ar" )) AND ( LIMIT-TO ( PUBYEAR, 2021) OR LIMIT-TO (PUBYEAR, 2020) } \\
\text { OR LIMIT-TO ( PUBYEAR, 2019) OR LIMIT-TO (PUBYEAR, 2018) OR LIMIT- } \\
\text { TO (PUBYEAR, 2017)) AND ( UMIT-TO (LANGUAGE, "English")) }\end{array}$ & 331 \\
\hline \multirow[t]{2}{*}{ Web of Science } & $\begin{array}{l}\text { (KP=( "Evolutionary computation" OR "Genetic algorithm*" OR "Evolutionary } \\
\text { algorithm*" OR "Genetic programming" OR "Metaheuristic*" OR "Meta } \\
\text { heuristic*") AND KP=( "Design" OR "Product design" OR "Product } \\
\text { development" OR "Computer Aided Design" OR "Quality function } \\
\text { deployment" OR "Finite element method") AND KP=("Multiobjective } \\
\text { optimization" OR "Multi-objective optimization" OR "Design optimization" } \\
\text { OR "Optimization")) AND IDIOMA: (English) AND TIPOS DE DOCUMENTO: } \\
\text { (Article) Tempo estipulado: Últimos } 5 \text { anos. }\end{array}$ & 1.183 \\
\hline & Total $=>$ & 1.748 \\
\hline
\end{tabular}

\subsection{Filtragem do banco de artigos}

A seguir realizou-se um filtro de redundância, excluindo os artigos repetidos através de um software próprio elaborado pelos autores. O banco de artigos brutos passou então a conter 1.524 trabalhos, cujos os títulos de todos esses artigos foram lidos no software Excel. Neste procedimento foram verificados quais os artigos que possuíam os títulos alinhados ao tema da pesquisa, sendo que apenas 244 artigos demonstraram satisfazer tal condição.

Os 244 artigos foram então ordenados, do maior para o menor, pelo percentual acumulado de citações. Na Figura 3.1 verifica-se o reconhecimento científico dos artigos com título alinhado ao tema de pesquisa, onde foi aplicada a regra de Pareto e observa-se a linha de corte em 85 artigos com 80,2\% das citações e os 159 artigos restantes com 19,8\% das citações acumuladas.

Desta forma, 85 artigos possuíam reconhecimento científico, compondo assim o Repositório $\mathrm{K}$, enquanto os demais 159 artigos, com reconhecimento científico não confirmado, compuseram o Repositório P. Através da leitura dos resumos do Repositório K, verificou-se que 39 artigos estavam alinhados ao tema, resultando no Repositório A. 


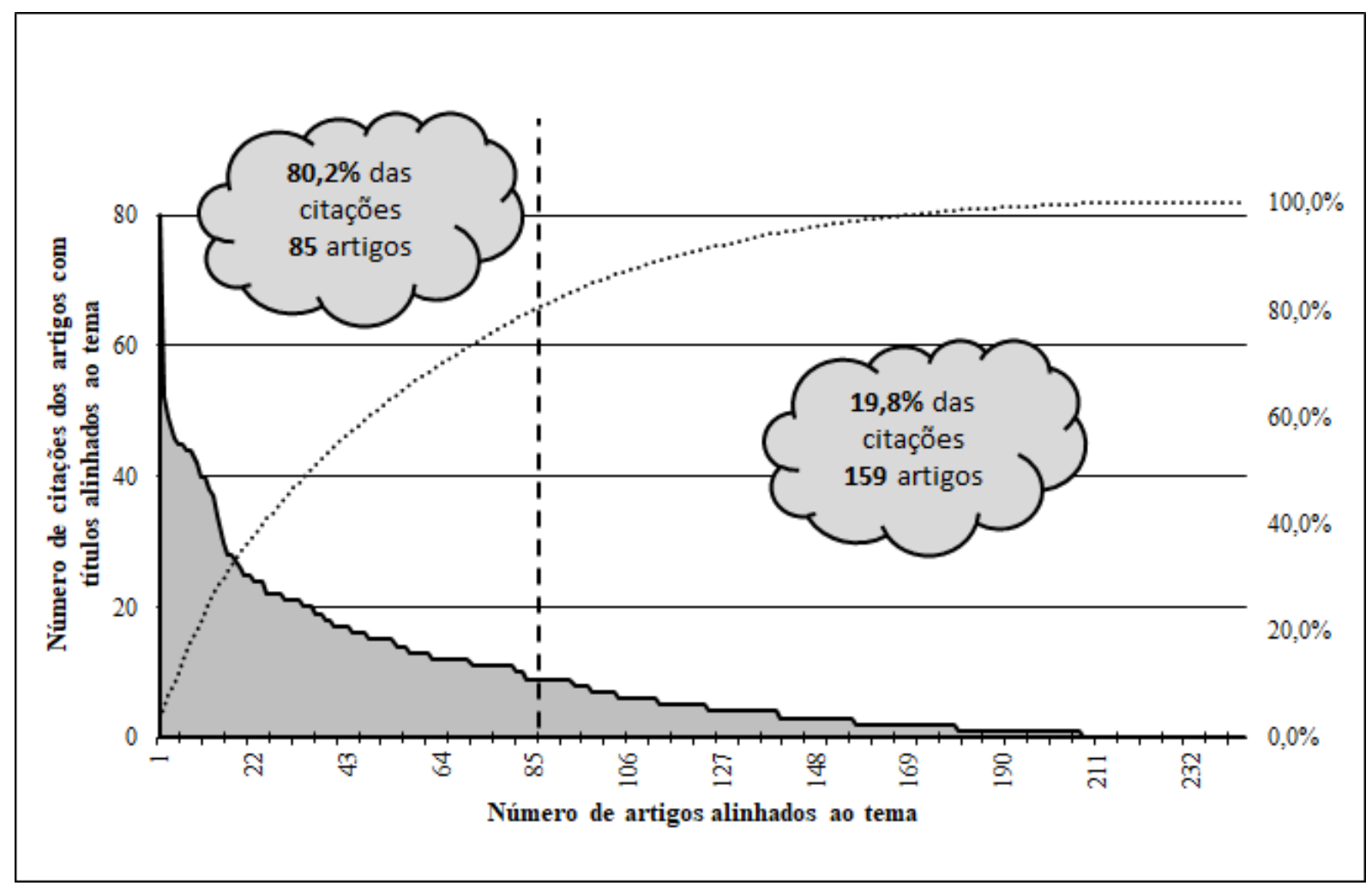

FIGURA 3.1 - Reconhecimento científico dos artigos com título alinhado ao tema de pesquisa. Fonte: Autoria própria

Dentro do Repositório $\mathrm{P}$ foram identificados 62 artigos recentes (publicados nos últimos 2 anos) e 12 artigos publicados por autores que também estavam no Repositório K. Foi lido então o resumo destes 74 artigos, resultando no Repositório B com 31 artigos. Através da soma dos Repositórios A e B obteve-se o Repositório C com 70 artigos.

Em seguida foi realizado o download dos artigos do Repositório C, sendo que 14 artigos não estavam disponíveis nas BDs. 56 artigos baixados foram lidos integralmente. Durante esta leitura, 6 artigos foram eliminados por não estarem alinhados ao tema, restando portanto 50 artigos. Para reduzir a quantidade de artigos para a análise sistêmica <40 artigos e ter um portfólio final altamente relevante, foi feito o levantamento dos fatores de impacto JCR, SNIP e classificação dos Quartis (Q1, Q2, Q3, Q4) dos periódicos dos 50 artigos. Se uma revista pertence ao Q1 (primeiro quartil), significa que ela possui um desempenho melhor do que pelo menos $75 \%$ das revistas da sua categoria. Foram então selecionadas apenas as publicações do Q1, obtendo-se o portfólio bibliográfico final de 32 artigos que pode ser verificado no Quadro 3.2. 
QUADRO 3.2 - Portfólio bibliográfico final. Fonte: Autoria própria.

\begin{tabular}{|c|c|c|c|c|}
\hline Cód. & Titulo do Artigo & Ano & AU & Citaçōes \\
\hline 1074 & $\begin{array}{l}\text { Economic opt imization of shell-and-tube heat exchanger using Jaya algorithm with } \\
\text { maintenance consideration }\end{array}$ & 2017 & Rao, RV; Saroj, A & 44 \\
\hline 1459 & $\begin{array}{l}\text { In-Wheel Switched Reluctance M otor Design for Electric Vehides by Using a Pareto- } \\
\text { Based Multiobjective Differ ential Evolution Algorithm }\end{array}$ & 2017 & Oksüztepe E. & 42 \\
\hline 1386 & $\begin{array}{l}\text { Optimal design of a seg mented thermoelectric generator based on three- } \\
\text { dimensianal numerical simulation and multi-objective genetic algorit hm }\end{array}$ & 2018 & Ge Y., Liu Z., Sun H., Liu W. & 37 \\
\hline 1366 & Design aptimization of battery pack endosure for el ectric vehicle & 2018 & $\begin{array}{l}\text { Shui L, Chen F., Garg A., } \\
\text { Peng X., Bao N., Zhang J. }\end{array}$ & 30 \\
\hline 1423 & $\begin{array}{l}\text { Simultaneous Optimization of Geometry and Firing Angles for In-Wheel Switched } \\
\text { Reluctance Motor Drive }\end{array}$ & 2018 & $\begin{array}{l}\text { Anvari B., Tolinat H.A., } \\
\text { Fahimi B. }\end{array}$ & 28 \\
\hline 1414 & Multiobjective Optimization of Five-Phase Induction Machines Based an NSGA-II & 2017 & $\begin{array}{l}\text { Pereira L.A., Haffner S, } \\
\text { Nical G., Dias T.F. }\end{array}$ & 25 \\
\hline 815 & $\begin{array}{l}\text { Multi-par ameter optimization of shell-and-tube heat exchanger with helical baffles } \\
\text { based on entrarsy theory }\end{array}$ & 2018 & $\begin{array}{l}\text { Wen, } \text {; Gu, X; Wang, MM; } \\
\text { Liu, YC; Wang, SM }\end{array}$ & 24 \\
\hline 1340 & A multiple lifecyde-based approach to sustainable product configuration design & 2018 & $\begin{array}{l}\text { Badurdeen F., Aydin R., } \\
\text { Brawn A. }\end{array}$ & 24 \\
\hline 1440 & $\begin{array}{l}\text { Integr ated design technique for materials and structures of vehicle body under } \\
\text { crash safety considerations }\end{array}$ & 2017 & $\begin{array}{l}\text { Chen Y., Liu G., Zhang Z., } \\
\text { Hou S. }\end{array}$ & 22 \\
\hline 1470 & $\begin{array}{l}\text { Multidisciplinary design optimization to ident ify additive manufacturing resources } \\
\text { in custornized product development }\end{array}$ & 2017 & Yaa X., Moon S.K., Bi G. & 22 \\
\hline 1214 & $\begin{array}{l}\text { Multiabjective System Level O ptimizat ion Method for Switched Reluctance Motor } \\
\text { Drive Systerns Using Finite-Element Model }\end{array}$ & 2020 & $\begin{array}{l}\text { Diao K., Sun X., Lei G., Guo } \\
\text { Y., Zhu J. }\end{array}$ & 20 \\
\hline 1046 & Aerodynamic-structural missile fin optimization & 2017 & $\begin{array}{l}\text { Vidanovic, N; Rasuo, B; } \\
\text { Kastratovic, G; Maksimavic, } \\
\text { S; Curcic, D; Sarnardzic, M }\end{array}$ & 18 \\
\hline 1439 & $\begin{array}{l}\text { Crushing analysis and multiobjective optimization design for rectangular unequal } \\
\text { triple-cell tubes subjected to axial loading }\end{array}$ & 2017 & $\begin{array}{l}\text { Shen W., Gu X., liang P., Hu } \\
\text { J., Lv X., Qian L }\end{array}$ & 17 \\
\hline 1449 & $\begin{array}{l}\text { Configuration apt imization of shell-and-tube heat exchangers with helical baffles } \\
\text { using multi-abjective genetic algorithm based an fluid-structure inter action }\end{array}$ & 2017 & $\begin{array}{l}\text { Wang S., Xiao J., Wang } \perp . \\
\text { Jian G., Wen } 1 ., \text { Zhang Z. }\end{array}$ & 16 \\
\hline 1424 & $\begin{array}{l}\text { Optimal design of a double-vibr ator ultr asoric motor using combination method of } \\
\text { finite element method, sersitivity analysis and adapt ive genetic a lgorithm }\end{array}$ & 2017 & Dong Z., Yang M. & 14 \\
\hline 520 & $\begin{array}{l}\text { Optimization investigation an configuration parameters of sine wavy fin in plate-fin } \\
\text { heat exchanger based on fluid structure interaction analysis }\end{array}$ & 2019 & $\begin{array}{l}\text { Wen, } 1 ; \mathrm{Li}, \mathrm{K} \text {; Wang, CL; } \\
\text { Zhang, } \mathrm{X} \text {; Wang, SM }\end{array}$ & 12 \\
\hline 1270 & $\begin{array}{l}\text { Design optimization of composite submerged cylindrical pressure hull using genetic } \\
\text { algorithm and finite element analysis }\end{array}$ & 2019 & $\begin{array}{l}\text { Imran M., Shi D., Torg L., } \\
\text { Waqas H.M. }\end{array}$ & 12 \\
\hline 1338 & $\begin{array}{l}\text { Cosging torque reduction of per manent magnet synchr onous motor using multi- } \\
\text { objective optimization }\end{array}$ & 2018 & $\begin{array}{l}\text { Ilka R., Alinejad-Ber omiY., } \\
\text { Yaghobi H. }\end{array}$ & 12 \\
\hline 1369 & $\begin{array}{l}\text { Multiobjective Optimization of a Shell-Like Induction Spherical Motor for a Power- } \\
\text { Assisted Wheekchair }\end{array}$ & 2018 & $\begin{array}{l}\text { Fernandes J.F.P., Vieira } \\
\text { S.M., Costa Branco PJ. }\end{array}$ & 12 \\
\hline 1273 & $\begin{array}{l}\text { Thermal optimization of a kirigami-patterned wear able lithium-ion battery based } \\
\text { on a navel design of camposite phase change material }\end{array}$ & 2019 & $\begin{array}{l}\text { Yang } M_{.}, \text {Wang } H_{.} \text {, Shuai } W_{\text {, }} \\
\text { Deng. }\end{array}$ & 11 \\
\hline 258 & $\begin{array}{l}\text { Optimal sizing of a wind, fuel cell, electr olyzer, battery and super capacitor system } \\
\text { for off-grid applications }\end{array}$ & 2020 & $\begin{array}{l}\text { Atternene, NS; Agbli, KS; } \\
\text { Fofana, S; Hissel, D }\end{array}$ & 9 \\
\hline 1271 & $\begin{array}{l}\text { Multi-objective and multi-constraint design optimization for hat-shaped composite } \\
\text { T-joints in autarnobiles }\end{array}$ & 2019 & $\begin{array}{l}\text { Hou W., Xu X., Han X., Wang } \\
\text { H., Tong L. }\end{array}$ & 8 \\
\hline 1415 & $\begin{array}{l}\text { Design Optimization of a Shart-Term Duty Electrical Machine for Extreme } \\
\text { Environment }\end{array}$ & 2017 & $\begin{array}{l}\text { Arumugain P., Amankwaht } \\
\text { E., Walker A., Gerada C. }\end{array}$ & 6 \\
\hline 1241 & $\begin{array}{l}\text { Multi-abjective aerodynamic and structural int egrated optimization design of wind } \\
\text { turbines at the system level through a coupled blade-tower madel }\end{array}$ & 2020 & Zhu J., Zhou Z., CaiX. & 4 \\
\hline 183 & $\begin{array}{l}\text { Application of genetic algorithm for optimization of control rods positioning in a } \\
\text { fast breeder reactor core }\end{array}$ & 2020 & $\begin{array}{l}\text { Ramachandran, S; Jayalal, } \\
\text { ML; Riyas, A; Jehadeesan, R; } \\
\text { Devan, } K\end{array}$ & 3 \\
\hline 65 & $\begin{array}{l}\text { An int egrated multi-objective opt imization method with applica tion to train } \\
\text { crashworthiness design }\end{array}$ & 2021 & $\begin{array}{l}\text { Hou, L; Zhang, HH; Peng, Y; } \\
\text { Wang, SM; Yao, S; Li, ZX; } \\
\text { Deng, GX }\end{array}$ & 2 \\
\hline 176 & Multi-objective opt imization of crastworthiness of vehicle front longitudinal bearn & 2020 & Wang, HY; Xie, H & 1 \\
\hline 71 & Madeling and Optimizing Method for Axial Flux Induction Motor of Electric Vehicles & 2020 & $\begin{array}{l}\text { Mei, J; Zuo, YF; Lee, CHT; } \\
\text { Kirtley, JL }\end{array}$ & 0 \\
\hline 265 & $\begin{array}{l}\text { Evolutionary algorithrns and the Preference Ranking Organization Method for } \\
\text { Enrichment Evaluations as applied to a multiobjective design of shell-and-tube heat } \\
\text { exchangers }\end{array}$ & 2020 & $\begin{array}{l}\text { Saldanha, WH; Arrieta, FRP; } \\
\text { Machado-Coelho, TM; dos } \\
\text { Santos, ED; Maia, CB; Ekel, } \\
\text { PI; Saares, GL. }\end{array}$ & 0 \\
\hline 1229 & $\begin{array}{l}\text { Integr ating a constr aint-based aptimization approach into the design of oil } \& \text { gas } \\
\text { structures }\end{array}$ & 2020 & $\begin{array}{l}\text { Cicconi P., Nardelli M., } \\
\text { Raffaeli R., Germani M. }\end{array}$ & 0 \\
\hline 1524 & Multi-objective apt imization of hypoid gears to improve operating characteristics & 2020 & Siman, Vilmos $\mathrm{V}$. & 0 \\
\hline 1188 & $\begin{array}{l}\text { Multiobjective Optimization of Inductive Power Transfer Double-D Pads for Electric } \\
\text { Vehicles }\end{array}$ & 2021 & $\begin{array}{l}\text { Luo Z., Wei X., Pearce } \\
\text { M.G.S., Covic G.A. }\end{array}$ & 0 \\
\hline
\end{tabular}




\section{ANÁLISE BIBLIOMÉTRICA}

Em seguida, após a seleção do portfólio bibliográfico (PB) foi realizada então a análise bibliométrica de forma a evidenciar quantitativamente dados estatísticos sobre os artigos selecionados. Para isso, quatro aspectos foram avaliados: (i) a evolução do tema; (ii) o grau de relevância dos periódicos; (iii) o fator de impacto JCR e SNIP dos periódicos; (iv) a relevância dos artigos e (v) as palavras-chave mais utilizadas.

Quanto à evolução do tema, na Figura 4.1 observa-se que a maior quantidade de artigos do portfólio bibliográfico publicados no tema foi no ano de 2017, com 10 artigos publicados em 2020, ainda se notando uma tendência da publicação de estudos relacionados com a temática.

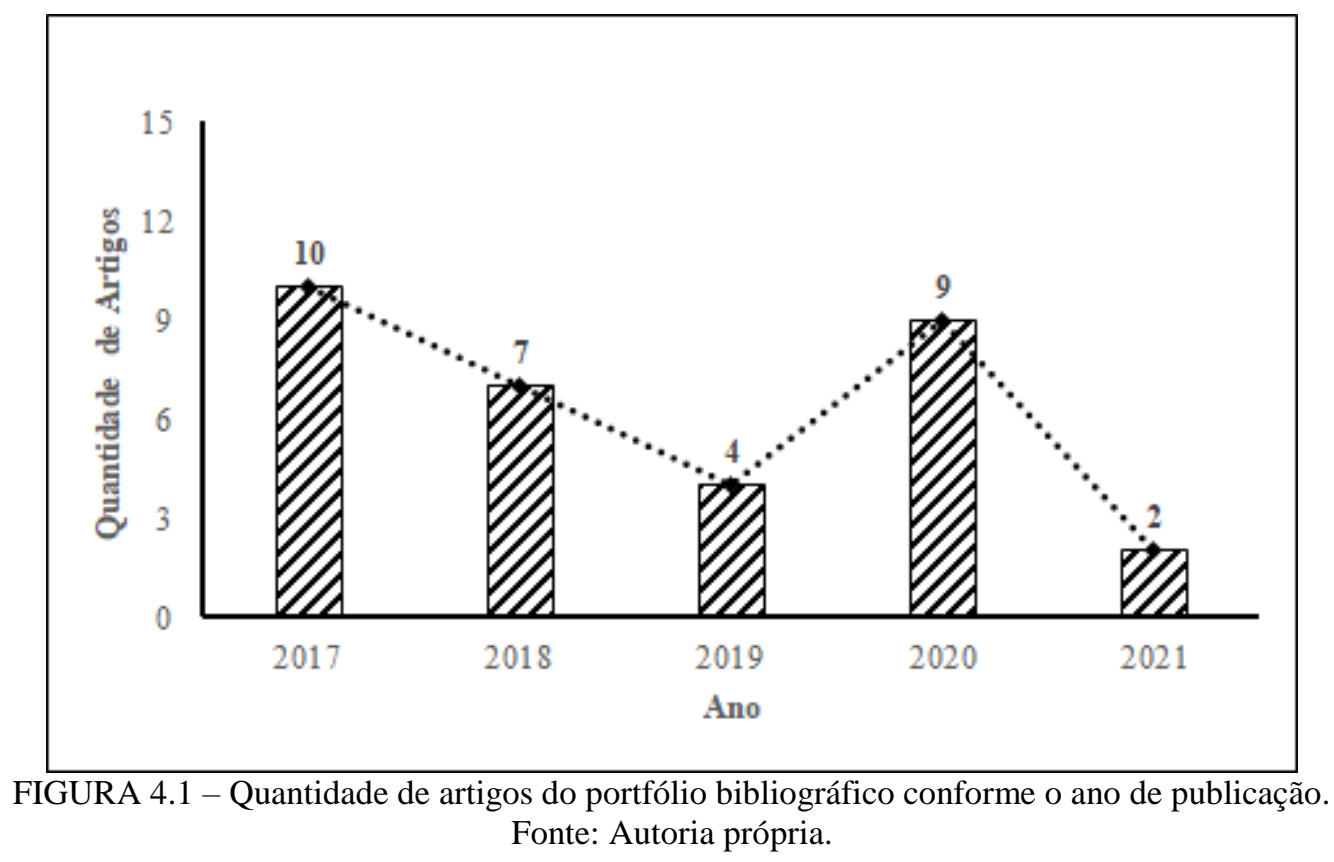

Quanto ao grau de relevância dos periódicos, observa-se na Figura 4.2 que o "Structural and Multidisciplinary Optimization”, contendo 4 artigos do portfólio em suas publicações, o "IEEE Transactions on Industrial Eletronics" e "Applied Thermal Engineering", com 3 artigos cada respectivamente. Quanto ao fator de impacto dos periódicos, ainda na Figura 4.2, o destaque fica para o "IEEE Transactions on Industrial Eletronics", cujo o JCR foi de 7,515 e SNIP de 3,292, os maiores entre todos periódicos da amostra. 


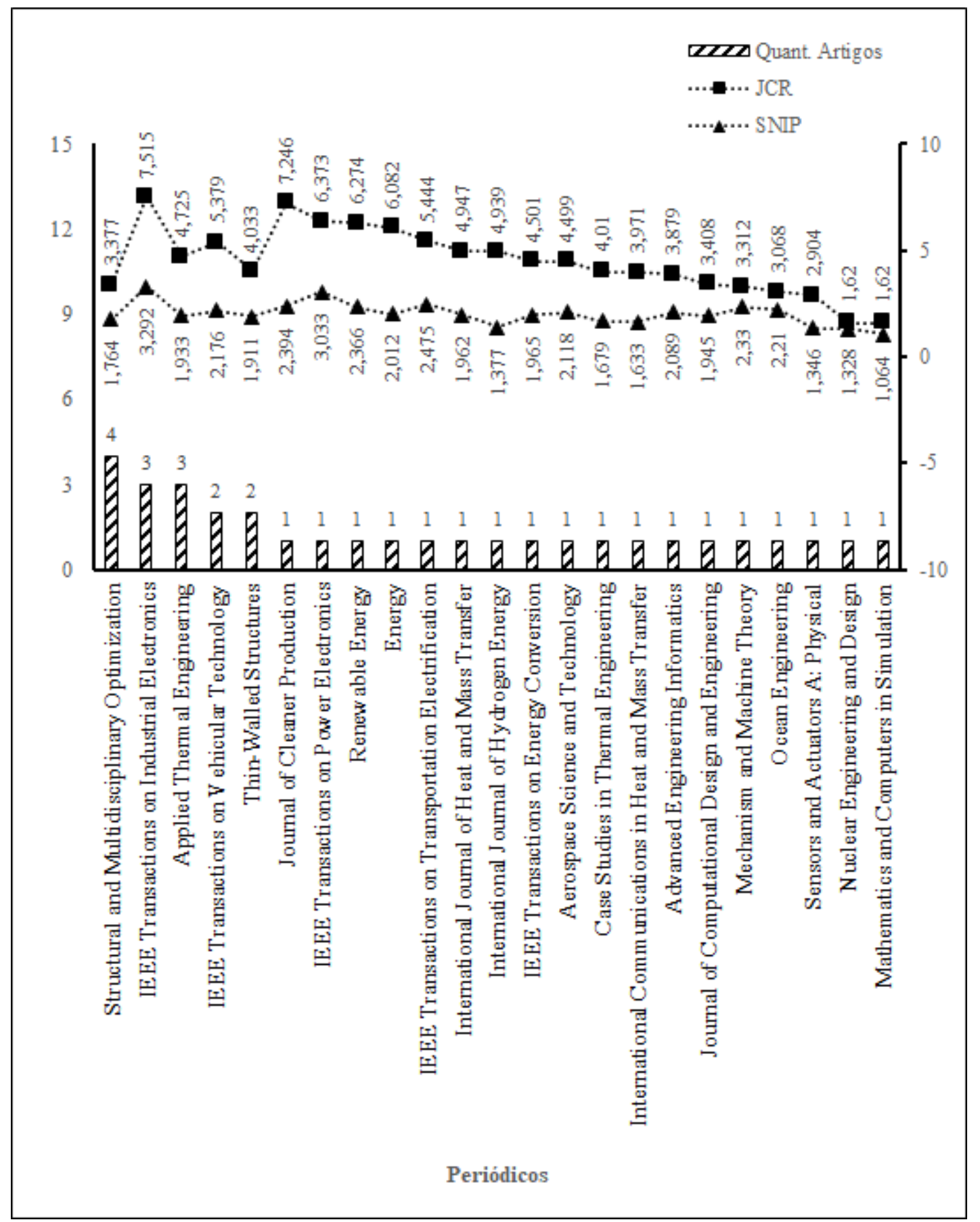

FIGURA 4.2 - Grau de relevância dos periódicos no portfólio bibliográfico. Fonte: Autoria própria.

Conforme a Figura 4.3, os artigos com mais relevância no PB foram os de Rao \& Saroj (2017) com 44 citações, Öksüztepe (2017) com 42 citações, Ge et al. (2018) com 37 citações e Shui et al. (2018) com 30 citações. 


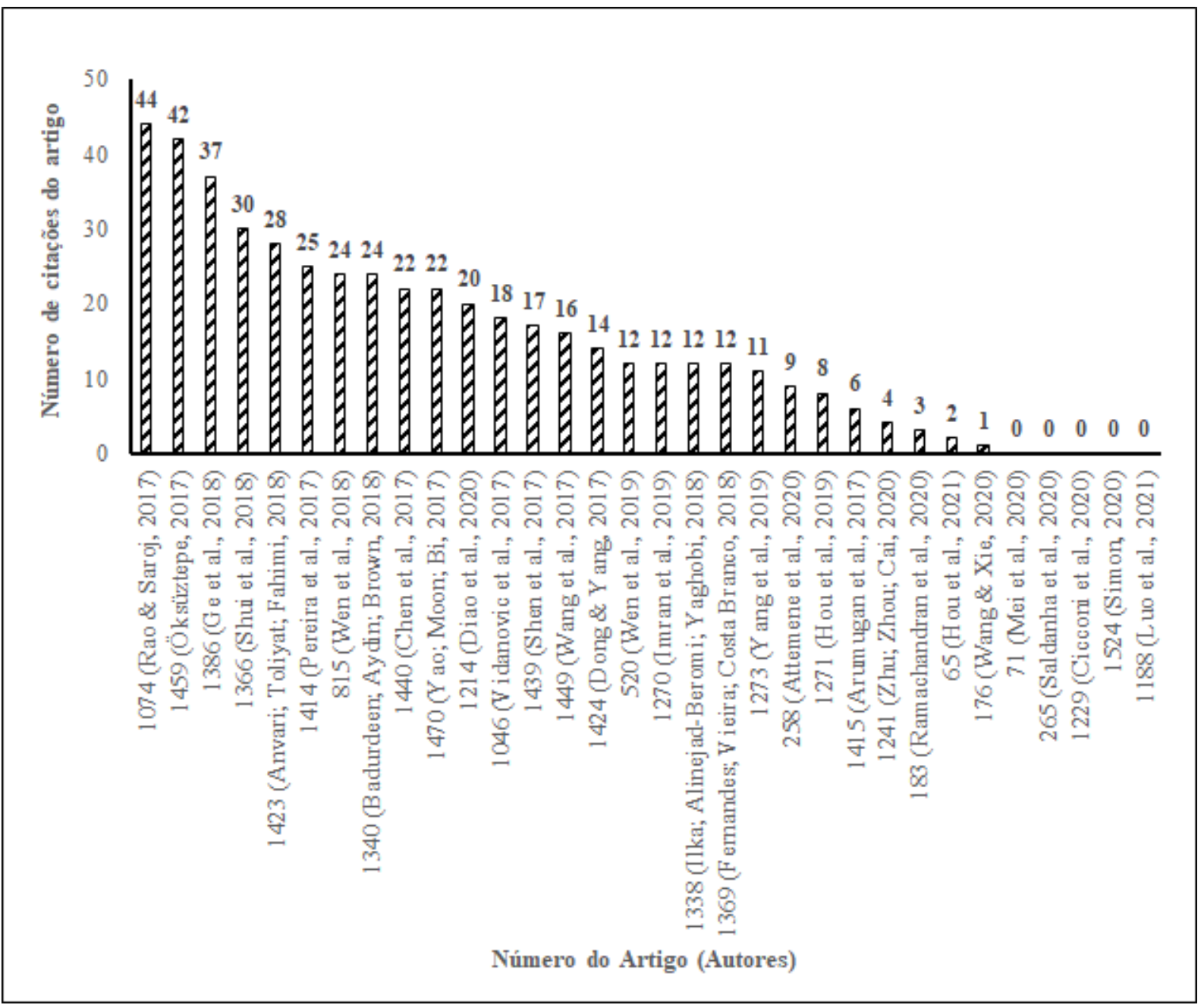

FIGURA 4.3 - Relevância dos artigos do portfólio bibliográfico. Fonte: Autoria própria.

De acordo com a Figura 4.4, as palavras-chave mais utilizadas no portfólio bibliográfico foram "Multi-objetctive optimization" com 13 utilizações, "Genetic algorithm" com 8 utilizações, "Optimization" com 5 utilizações e "Design Optimization" com 4 utilizações. 


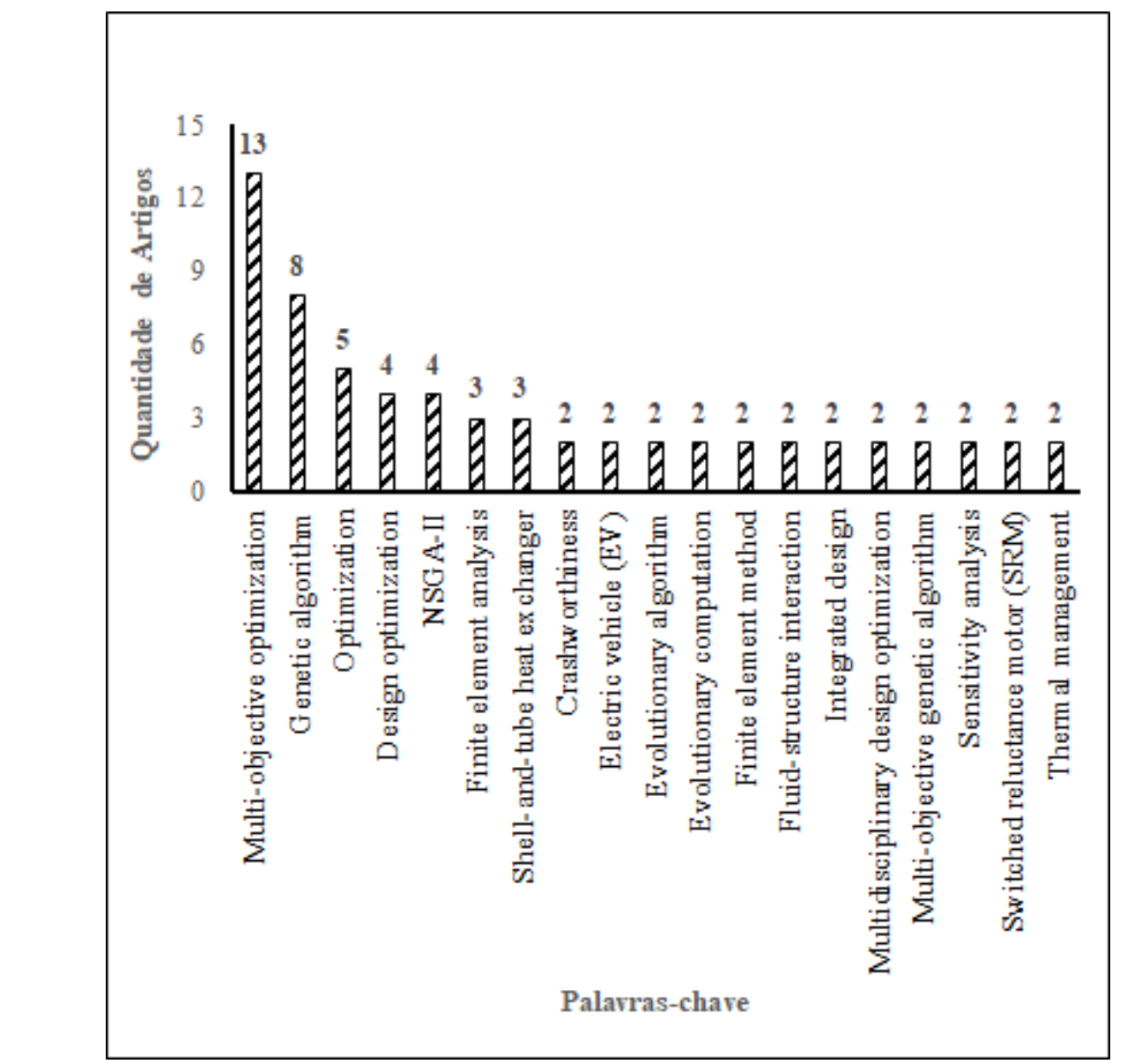

FIGURA 4.4 - Palavras-chave mais utilizadas no portfólio bibliográfico. Fonte: Autoria própria.

\section{ANÁLISE SISTÊMICA}

Nesta etapa foi analisado o conteúdo dos 32 artigos selecionados no portfólio final, verificando-se: (i) quais foram os problemas de pesquisa dos trabalhos; (ii) quais os objetivos e recursos propostos pelos autores para solucionar esses problemas; e (iii) quais as lacunas existentes, as limitações e as recomendações para trabalhos futuros feitas pelos autores.

\subsection{Problemas de pesquisa}

Os artigos selecionados apresentam problemas de otimização multiobjetivo no design de 15 tipos de produtos utilizando algum tipo de técnica de CE. No Quadro 5.1 verifica-se que os estudos se concentraram em motores elétricos, trocadores de calor, carrocerias de automóveis, turbinas eólicas e baterias de íons de lítio. 
QUADRO 5.1 - Problemas de pesquisa por autor e tipos de produtos. Fonte: Autoria própria.

\begin{tabular}{|c|c|c|}
\hline Quant. & Autores & Problema/Produto \\
\hline 9 & $\begin{array}{l}\text { Arumugan et al. (2017); Dong \&. Yang (2017); Öksüztepe (2017); Pereira et } \\
\text { al. (2017); Anvari, Toliyat \&. Fahimi (2018); Ilka, Alinejad-Beromi \& Yaghobi } \\
\text { (2018); Femandes, Vieira \& Costa Branco (2018); Diao et al. (2020); Mei et } \\
\text { al. (2020). }\end{array}$ & Motores elétricos \\
\hline 5 & $\begin{array}{l}\text { Rao \& Saroj (2017); Wen et al. (2018); Wang et al. (2017); Wen et al. } \\
\text { (2019); Saldanha et al. (2020). }\end{array}$ & Trocadores de calor \\
\hline 4 & Chen et al. (2017); Shen et al. (2017); Hou et al. (2019); Wang \& Xie (2020) & Carroceria de automóveis \\
\hline 2 & Attemene et al. (2020) e Zhu, Zhou \& Cai (2020) & Turbinas eólicas \\
\hline 2 & Shui et al. (2018) e Yang et al. (2019) & Bateria de ions de lítio \\
\hline 1 & Badurdeen, Aydin \& Brown (2018) & Cartucho de toner \\
\hline 1 & Cicconi et al. $(2020)$ & $\begin{array}{l}\text { Projeto de uma estrutura de aço de } 700 \\
\text { toneladas para Óleo \& Gás }\end{array}$ \\
\hline 1 & Ge et al. (2018) & Gerador termoelétrico segmenta do (TEG) \\
\hline 1 & Hou et al. (2021) & Chassi de um trem \\
\hline 1 & Imran et al. (2019) & Casco de pressão de submersivel \\
\hline 1 & Luo et al. (2021) & Bobina de indução Double-D \\
\hline 1 & Ramachandran et al. (2020) & Reator nuclear \\
\hline 1 & Simon $(2020)$ & Engrenagens hipóides \\
\hline 1 & Vidanovic et al. (2017) & Míssil balistico de curto alcance \\
\hline 1 & Yao, Moon \& Bi (2017) & $\begin{array}{l}\text { Próteses transtibiais customiz adas por } \\
\text { manufatura aditiva }\end{array}$ \\
\hline
\end{tabular}

\subsection{Objetivos e recursos propostos}

Quanto aos objetivos e recursos propostos pelos autores para resolverem os problemas de otimização, como observa-se no Quadro 5.2, o principal recurso utilizado foi o algoritmo genético de classificação por não dominância II (NSGA-II) com 15 artigos, seguido pelos algoritmos genéticos multiobjetivo (MOGA) e algoritmos genéticos (clássicos).

QUADRO 5.2 - Objetivos e recursos propostos nas pesquisas. Fonte: Autoria própria.

\begin{tabular}{|c|c|c|}
\hline Quant. & Autores & Objetivos/Recursos \\
\hline 15 & $\begin{array}{l}\text { Shui et al. (2018); Anvari, Toliyat \& Fahimi (2018); Pereira et al. } \\
\text { (2017); Badurdeen, Aydin \& Brown ( 2018); Chen et al. (2017); } \\
\text { Shen et al. (2017); llka, Alinejad-Beromi \& Yaghobi (2018); } \\
\text { Fernandes, Vieira \& Costa Branco (2018); Attemene et al. } \\
\text { (2020); Hou et al. (2019); Arumugan et al. (2017); Zhu, Zhou \& } \\
\text { Cai (2020); Wang \& Xie (2020); Luo et al. (2021); Simon (2020) }\end{array}$ & $\begin{array}{l}\text { Aplicação do algoritmo genético classificado não } \\
\text { dominado (NSGA II) }\end{array}$ \\
\hline 5 & $\begin{array}{l}\text { Vidanovic et al. (2017); Yao, Moon \& Bi (2017); Wang et al. } \\
\text { (2017); Ge et al. (2018); Wen et al. (2019) }\end{array}$ & Uso de algoritmos genéticos multiobjetivo (MOGA) \\
\hline 5 & $\begin{array}{l}\text { Wen et al. (2018); Diao et al. (2020); Imran et al. (2019); } \\
\text { Ramachandran et al. (2020); Mei et al. (2020) }\end{array}$ & Uso de algoritmo genéticos (clássicos) \\
\hline 1 & Cicconi et al. $(2020)$ & Uso do algoritmo backtracking \\
\hline 1 & Dong \& Yang (2017) & Uso do algoritmo genético adaptativo \\
\hline 1 & Hou et al. (2021) & $\begin{array}{l}\text { Aplicação do algoritmo evolutivo multi-objetivo } \\
\text { baseado em indicador (AR-MOEA) }\end{array}$ \\
\hline 1 & Öksūztepe (2017) & $\begin{array}{l}\text { Uso do algoritmo de evolução diferencial } \\
\text { multiobjetivo basea do em Pareto (PMODEA) }\end{array}$ \\
\hline 1 & Rao \& Saroj (2017) & $\begin{array}{l}\text { Comparação de usodo algoritmo Jaya com } \mathrm{GA}, \mathrm{PSO} \text { e } \\
\text { CSO }\end{array}$ \\
\hline 1 & Saldanha et al. (2020) & Comparação entre os algoritmos MO PSO e NSGA II \\
\hline 1 & Yang et al. (2019) & $\begin{array}{l}\text { Comparação do uso dos algoritmos MOPSO, NSGA-III } \\
\text { e SPEA-\|I }\end{array}$ \\
\hline
\end{tabular}




\subsection{Lacunas existentes}

No Quadro 5.3 são relacionadas as principais lacunas, limitações e recomendações dos estudos. Os autores sugerem principalmente a aplicação do método utilizado na pesquisa em outros sistemas, a incorporação de novos parâmetros de otimização no método utilizado, a aplicação do método atendendo outros critérios ou com diferentes métodos ou algoritmos ao mesmo sistema.

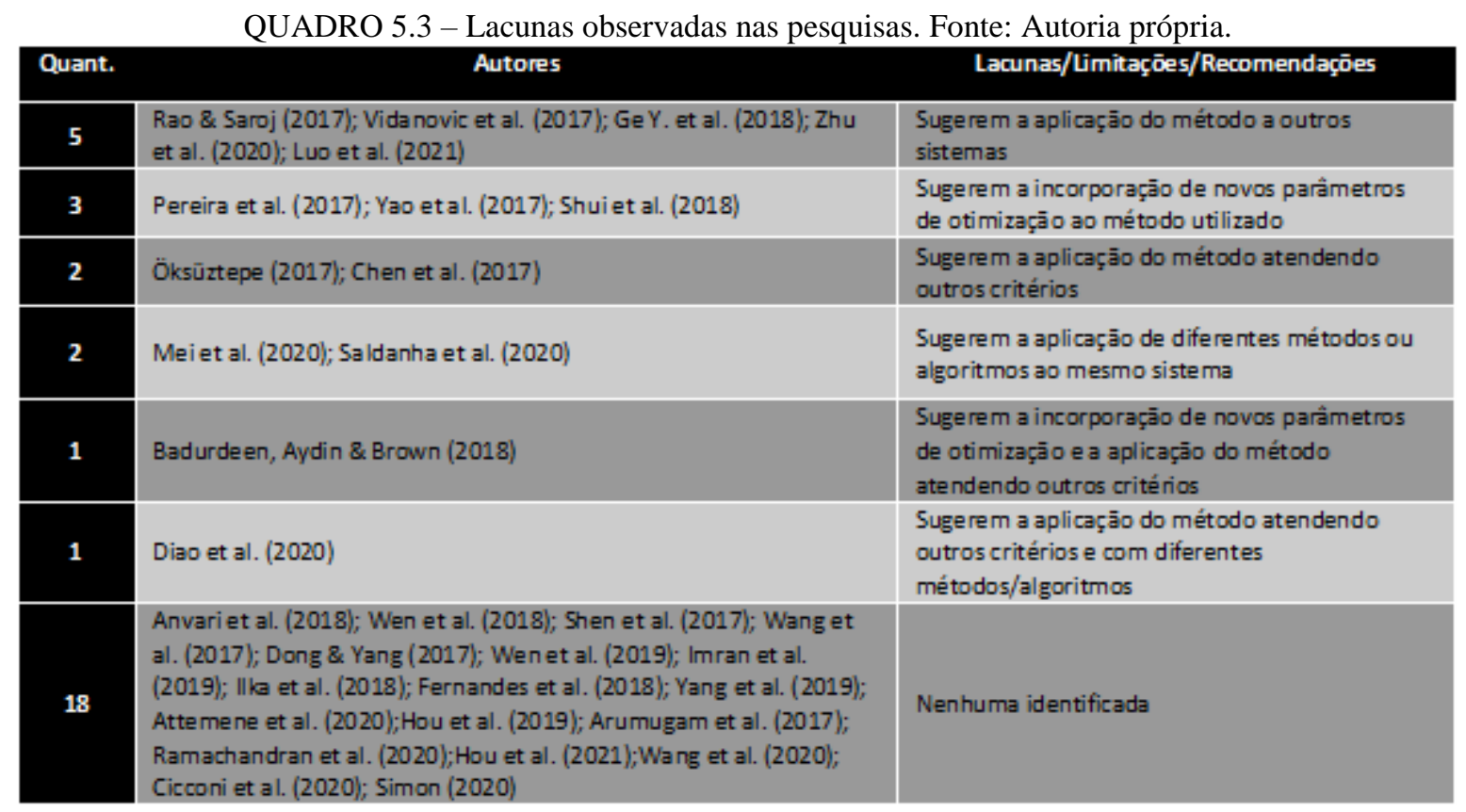

\section{CONCLUSÃO}

Este estudo realizou uma análise bibliográfica e sistêmica sobre o uso da otimização multiobjetivo no design de produtos através de uma metodologia estruturada denominada ProKnow-C. Primeiramente foi criado o portfólio bibliográfico, a partir de 1.748 artigos encontrados em três bases de dados entre 2017 e 2021, onde 32 artigos relacionados ao tema foram selecionados. Em segundo lugar, uma análise bibliométrica dos artigos permitiu a avaliação do portfólio bibliográfico, por meio de gráficos comparativos quanto à relevância dos periódicos, o seu fator de impacto, o reconhecimento científico, ano de publicação, autores e palavras-chaves mais utilizadas. Por fim, foi realizada uma análise sistêmica com a leitura completa de todos os artigos selecionados para identificar os principais problemas de pesquisa, objetivos e recursos propostos e as lacunas existentes.

As principais tendências encontradas foram o emprego das técnicas de CE para a otimização de diversos tipos de produtos, destacando-se o uso para o design de motores elétricos, trocadores de calor, carrocerias de automóveis, turbinas eólicas e baterias de íons de lítio. A 
análise sistêmica indicou que pesquisas aplicando o algoritmo genético de classificação por não dominância II (NSGA-II) são as mais comuns, seguido pelos algoritmos genéticos multiobjetivo (MOGA) e algoritmos genéticos clássicos. Por fim, como oportunidades, as principais sugestões foram aplicar o método utilizado em outros sistemas e a incorporação de novos parâmetros de otimização ao método utilizado.

Este estudo limitou-se as três primeiras etapas do ProKnow-C. Verificou-se que esta metodologia foi eficaz para a compreensão dos problemas atuais, objetivos, lacunas e oportunidades sobre a temática. Devido ao grande número de artigos que são encontrados nas bases de dados foi necessário aplicar filtros adicionais para que o número de artigos fosse reduzido e ainda se mantivesse representativo para realizar as análises dentro de tempo disponível. Em estudos futuros, recomenda-se aprofundar as análises das referências utilizadas pelos autores do PB e também as cocitações, verificando quais artigos que serviram de base para os autores, não somente os autores recentes, mas aqueles que cunharam conceitos importantes para as pesquisas. Também sugere-se incluir na seleção os artigos de congressos.

\section{AGRADECIMENTOS}

Os autores agradecem à Fundação de Desenvolvimento da Pesquisa - Fundep - Rota 2030 pelo apoio para o desenvolvimento deste trabalho.

\section{REFERÊNCIAS}

ANVARI, B.; TOLIYAT, H.A.; FAHIMI, B. Simultaneous optimization of geometry and firing angles for inwheel switched reluctance motor drive. IEEE Transactions on Transportation Electrification, v. 4, n. 1,p. 322-329, March 2018.

ARUMUGAM, P.; AMANKWAH, E.; WALKER, A.; GERADA, C. Design optimization of a short-term duty electrical machine for extreme environment. IEEE Transactions on Industrial Electronics, v.64, n. 12, p. 9.784-9.794, Dec. 2017.

ATTEMENE, N.S.; AGBLI, K.S.; FOFANA, S.; HISSEL, D. Optimal sizing of a wind, fuel cell, electrolyzer, battery and supercapacitor system for off-grid applications. International Journal of Hydrogen Energy, v. 45, n. 8, p. 5.512-5.525, February 2020.

BÄCK, T.; HAMMEL, U.; SCHWEFEL, H. Evolutionary computation: comments on the history and current state. IEEE Transactions on Evolutionary Computation, v.1, n.1, April 1997.

BADURDEEN, F.; AYDIN, R.; BROWN, A. A multiple lifecycle-based approach to sustainable product configuration design. Journal of Cleaner Production, v. 200, p. 756-769, November 2018.

CHEN, Y.; LIU, G.; ZHANG, Z.; HOU, S. Integrated design technique for materials and structures of vehicle body under crash safety considerations. Structural and Multidisciplinary Optimization, v. 56, p. 455-472, 2017.

CICCONI, P.; NARDELLI, M.; RAFFAELI, R.; GERMANI, M. Integrating a constraint-based optimization approach into the design of oil \& gas structures. Advanced Engineering Informatics, v. 45, August 2020. 
COELHO, L.S.; MARIANI, V.C. Sistema híbrido neuro-evolutivo aplicado ao controle de um processo multivariável. Revista Controle \& Automação, v.17, n.1, p. 32-48, 2006.

DIAO, K.; SUN, X.; LEI, G.; GUO, Y.; ZHU, J. Multiobjective system level optimization method for switched reluctance motor drive systems using finite-element model. IEEE Transactions on Industrial Electronics, v. 67, n. 12, p. 10.055-10.064, Dec. 2020.

DONG, Z.; YANG, M. Optimal design of a double-vibrator ultrasonic motor using combination method of finite element method, sensitivity analysis and adaptive genetic algorithm. Sensors and Actuators A: Physical, v. 266, p. 1-8, October 2017.

ENSSLIN, L.; ENSSLIN, S.R.; LACERDA, R.; TASCA, J. ProKnow-C, knowledge development process construtivist. Processo técnico com patente de registro pendente junto ao INPÌ. 2010.

FERNANDES, J.F.P;, VIEIRA, S.M.; COSTA BRANCO, P.J. Multiobjective optimization of a shell-like induction spherical motor for a power-assisted wheelchair. IEEE Transactions on Energy Conversion, v. 33, n. 2, p. 660-669, June 2018.

GASPAR-CUNHA, A.; TAKAHASHI, R.; ANTUNES, C.H. Manual de computação evolutiva e metaheurística. Belo Horizonte: Editora UFMG, 2012.

GE, Y.; LIU, Z.; SUN, H.; LIU, W. Optimal design of a segmented thermoelectric generator based on threedimensional numerical simulation and multi-objective genetic algorithm. Energy, v. 147, p. 1060-1069, March 2018.

HOU, L; ZHANG, H.H.; PENG, Y.; WANG, S.M.; YAO, S.; LI, Z.X.; DENG, G.X. An integrated multiobjective optimization method with application to train crashworthiness design. Structural and Multidisciplinary Optimization, v. 63, p. 1.513-1.532, 2021.

HOU, W.; XU, X.; HAN, X.; WANG, H.; TONG, L. Multi-objective and multi-constraint design optimization for hat-shaped composite T-joints in automobiles. Thin-Walled Structures, v. 143, October 2019.

ILKA, R.; ALINEJAD-BEROMI, Y.; YAGHOBI, H. Cogging torque reduction of permanent magnet synchronous motor using multi-objective optimization. Mathematics and Computers in Simulation, v. 153, p. 83-95, November 2018.

IMRAN, M.; SHI, D.; TONG, L.; WAQAS, H.M. Design optimization of composite submerged cylindrical pressure hull using genetic algorithm and finite element analysis. Ocean Engineering, v. 190, October 2019.

LI, B.; HOU, B.; YU, W.; LU, X.; YANG, C. Applications of artificial intelligence in intelligent manufacturing: a review. Frontiers of Information Technology \& Electronic Engineering, v. 18, n. 1, p. 86-96, 2017.

LUO, Z.; WEI, X.; PEARCE, M.G.S.; COVIC, G.A. Multiobjective optimization of inductive power transfer double-d pads for electric vehicles. IEEE Transactions on Power Electronics, v. 36, n. 5, p. 5.135-5.146, May 2021.

MEI, J; ZUO, Y.F.; LEE, C.H.T.; KIRTLEY, J.L. Modeling and optimizing method for axial flux induction motor of electric vehicles. IEEE Transactions on Vehicular Technology, v. 69, n. 11, p. 12.822-12.831, Nov. 2020 .

ÖKSÜZTEPE, E. In-wheel switched reluctance motor design for electric vehicles by using a pareto-based multiobjective differential evolution algorithm. IEEE Transactions on Vehicular Technology, v. 66, n. 6, p. 4706-4715, June 2017.

PEREIRA, L.A.; HAFFNER, S.; NICOL, G.; DIAS, T.F. Multiobjective optimization of five-phase induction machines based on NSGA-II. IEEE Transactions on Industrial Electronics, v. 64, n. 12, p. 9844-9853, Dec. 2017.

RAMACHANDRAN, S.; JAYALAL, M.L.; RIYAS, A.; JEHADEESAN, R.; DEVAN, K. Application of genetic algorithm for optimization of control rods positioning in a fast breeder reactor core. Nuclear Engineering and Design, v. 361, May 2020.

RAO, R.V.; SAROJ, A. Economic optimization of shell-and-tube heat exchanger using Jaya algorithm with maintenance consideration. Applied Thermal Engineering, v. 116, p. 473-487, april 2017.

ROZENFELD, H.; FORCELliNI, F. A.; AMARAL, D. C.; TOLEDO, J. C.; SILVA, S. L.; ALLIPRANDINI, D. H.; SCALICE, R. K. Desenvolvimento de produtos: uma referência para a melhoria do processo. São Paulo: Editora Saraiva: 2012. 
SALDANHA, W.H.; ARRIETA, F.R.P.; MACHADO-COELHO, T.M; DOS SANTOS, E.D.; MAIA, C.B.; EKEL, P.I.; SOARES, G.L. Evolutionary algorithms and the preference ranking organization method for enrichment evaluations as applied to a multiobjective design of shell-and-tube heat exchangers. Case Studies in Thermal Engineering, v. 17, February 2020.

SHEN, W.; GU, X.; JIANG, P.; HU, J.; LV, X.; QIAN, L. Crushing analysis and multiobjective optimization design for rectangular unequal triple-cell tubes subjected to axial loading. Thin-Walled Structures, v. 117, p. 190-198, August 2017.

SHUI, L.; CHEN, F.; GARG, A.; PENG, X.; BAO, N.; ZHANG, J. Design optimization of battery pack enclosure for electric vehicle. Structural and Multidisciplinary Optimization, v. 58, p. 331-347, 2018.

SICHMAN, J.S. Inteligência Artificial e sociedade: avanços e riscos. Estudos Avançados, v. 35, n. 101, p-3744, 2021.

SIMON, V.V. Multi-objective optimization of hypoid gears to improve operating characteristics. Mechanism and Machine Theory, v. 146, April 2020.

VIDANOVIC, N.; RASUO, B.; KASTRATOVIC, G.; MAKSIMOVIC, S.; CURCIC, D.; SAMARDZIC, M. Aerodynamic-structural missile fin optimization. Aerospace Science and Technology, v. 65, p. 26-45, June 2017.

WANG, H.Y.; XIE, H. Multi-objective optimization of crashworthiness of vehicle front longitudinal beam. Structural and Multidisciplinary Optimization, v. 61, p. 2.111-2.123, 2020.

WANG, S.; XIAO, J.; WANG, J.; JIAN, G.; WEN, J.; ZHANG, Z. Configuration optimization of shell-and-tube heat exchangers with helical baffles using multi-objective genetic algorithm based on fluid-structure interaction. International Communications in Heat and Mass Transfer, v. 85, p. 62-69, July 2017.

WEN, J.; GU, X.; WANG, M.M.; LIU, Y.C.; WANG, S.M. Multi-parameter optimization of shell-and-tube heat exchanger with helical baffles based on entransy theory. Applied Thermal Engineering, v. 130, p. 804-813, February 2018.

WEN, J; LI, K; WANG, C.L.; ZHANG, X; WANG, S.M. Optimization investigation on configuration parameters of sine wavy fin in plate-fin heat exchanger based on fluid structure interaction analysis. International Journal of Heat and Mass Transfer, v. 131, p. 385-402, March 2019.

YANG, M.; WANG, H.; SHUAI, W.; DENG, X. Thermal optimization of a kirigami-patterned wearable lithium-ion battery based on a novel design of composite phase change material. Applied Thermal Engineering, v. 161, October 2019.

YAO, X.; MOON, S.K.; BI, G. Multidisciplinary design optimization to identify additive manufacturing resources in customized product development. Journal of Computational Design and Engineering, v. 4, n. 2, p. 131-142, April 2017.

ZHU, J.; ZHOU, Z.; CAI, X. Multi-objective aerodynamic and structural integrated optimization design of wind turbines at the system level through a coupled blade-tower model. Renewable Energy, v. 150, p. 523-537, May 2020. 